\title{
Hydroxyl Radical Generation Through the Fenton-like Reaction of Hematin- and Catechol-Functionalized Microgels
}

Pegah Kord Forooshani ${ }^{1}$, Rattapol Pinnaratip ${ }^{1}$, Elizabeth Polega ${ }^{1}$, Ariana G. Tyo ${ }^{1}$, Eric

Pearson ${ }^{2}$, Bo Liuํ․ Tinu-Ololade Folayan ${ }^{2}$, Lei Pan ${ }^{2}$, Rupak M. Rajachar ${ }^{1}$, Caryn L. Heldt ${ }^{2}$, Bruce P. Lee ${ }^{1 *}$

${ }^{1}$ Department of Biomedical Engineering, Michigan Technological University, Houghton, MI 49931, USA

${ }^{2}$ Department of Chemical Engineering, Michigan Technological University, Houghton, MI 49931, USA

*bplee@mtu.edu 


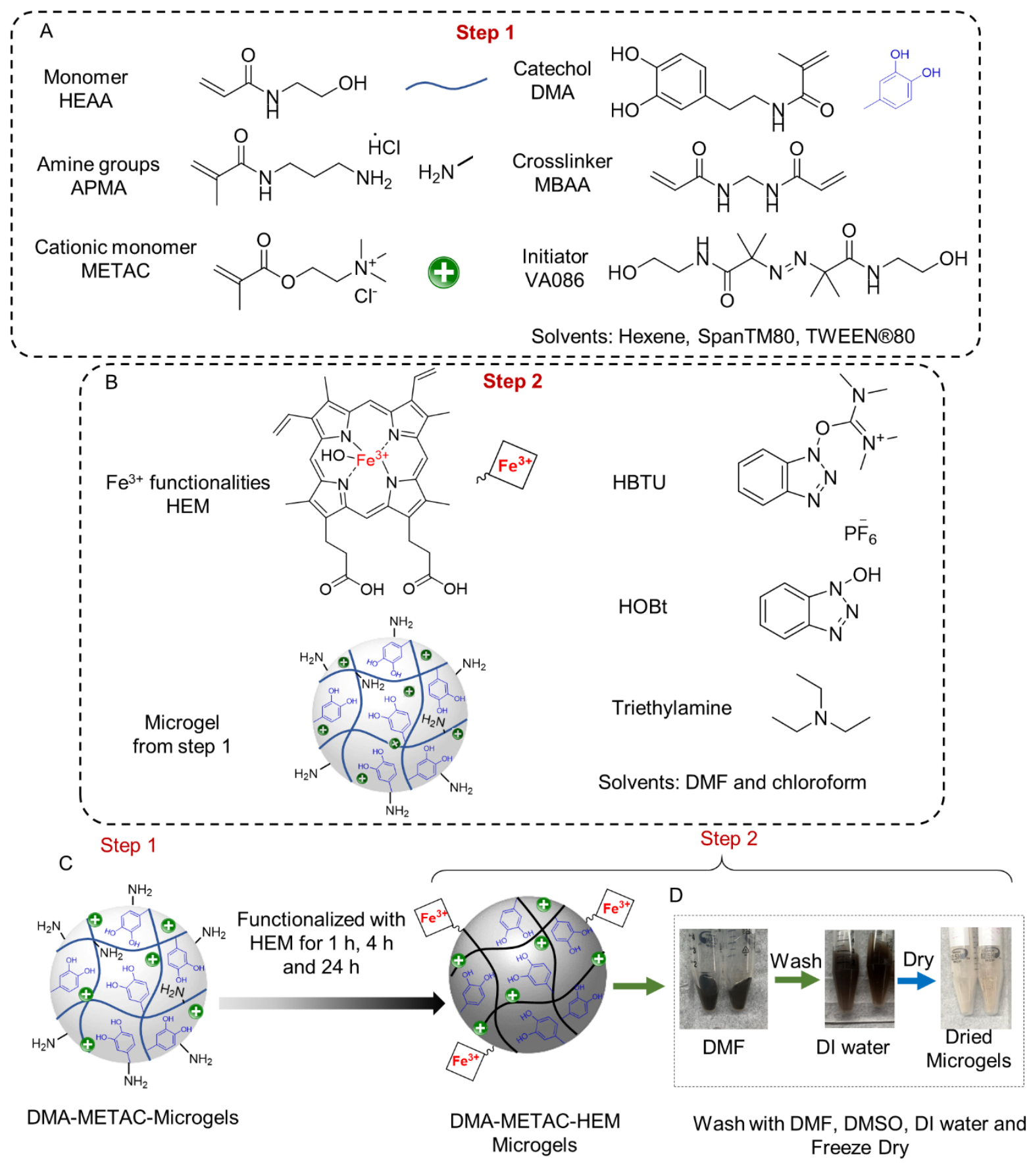

Figure S1. Preparation of HEM and DMA containing microgel in a two-step process. Chemical structure of monomers used to prepare DMA-containing microgels with APMA, an $-\mathrm{NH}_{2}$ containing monomer (A). Compounds and reactants used in the second step, to functionalizing APMA-functionalized microgels with HEM through carbodiimide chemistry (B) Schematic representation of the 2-step synthesis approach $(\mathrm{C})$. The resulted microgels were washed with DMF, DMSO, and DI water in succession and lyophilized to obtain dried microgels (D). 


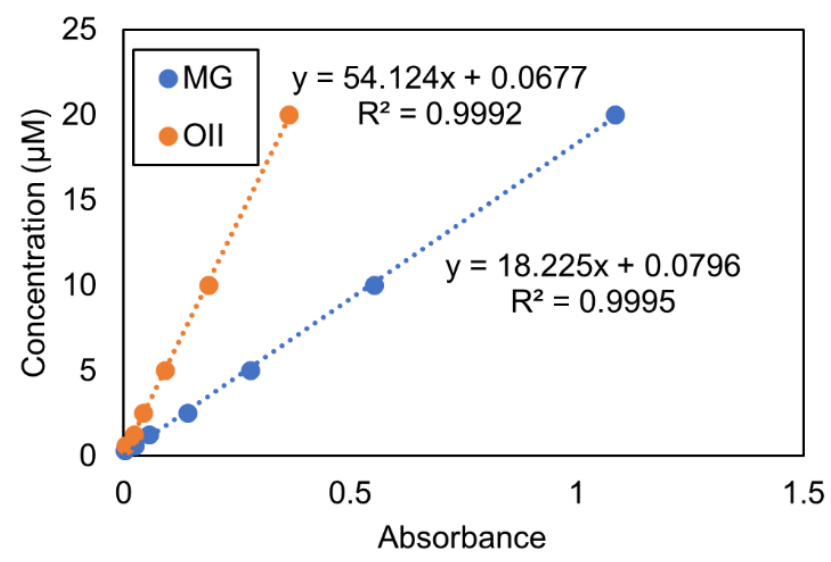

Figure S2. Standard curves for MG and OII dyes and their respective trend lines. The standard curves were prepared using a series of solutions containing $0-20 \mu \mathrm{M}$ of $\mathrm{MG}$ or OII. The concentration of the MG and OII was estimated using UV-vis at the wavelength of $617 \mathrm{~nm}$ and $484 \mathrm{~nm}$, respectively. 

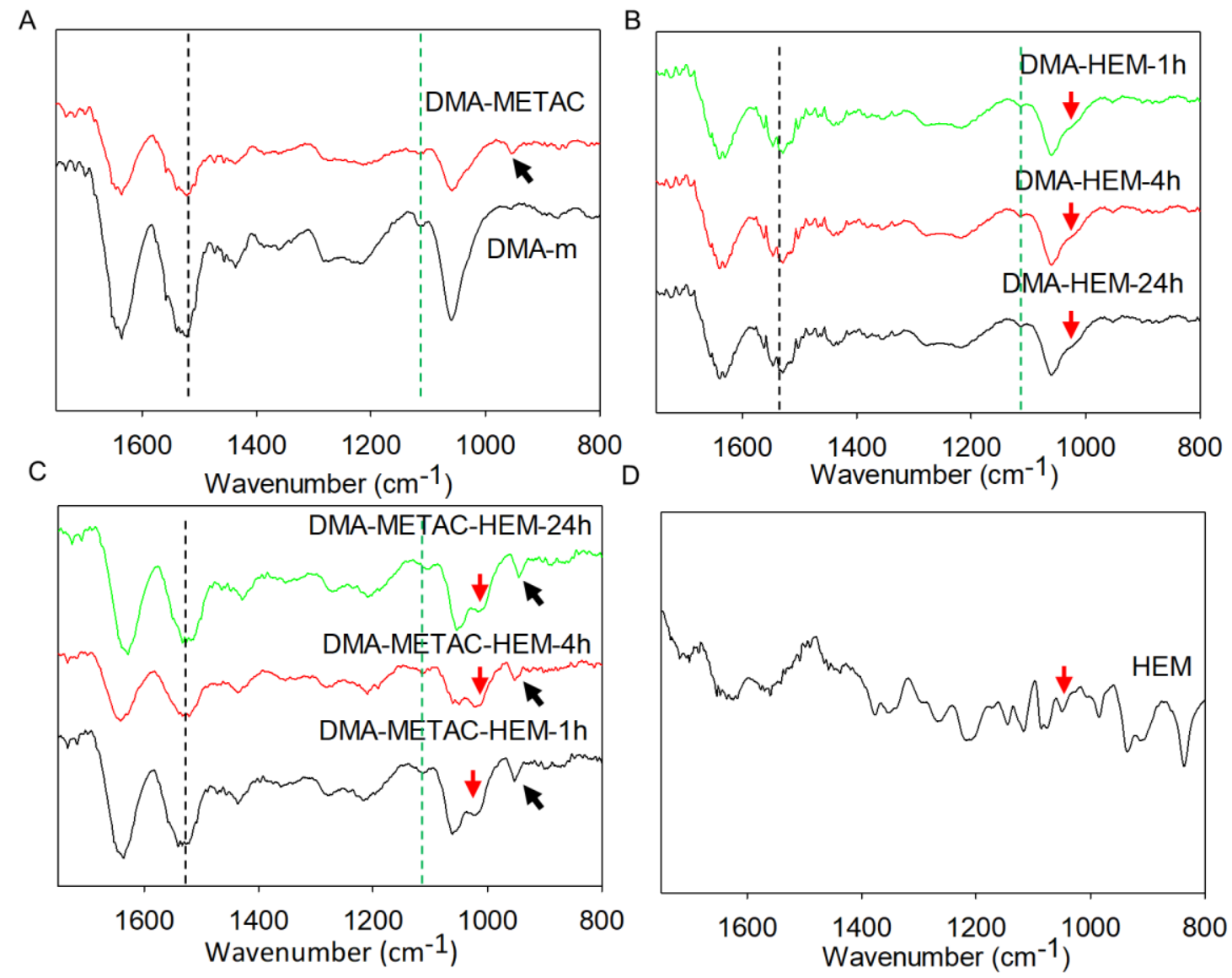

Figure S3. ATR-FTIR spectra of DMA-m and DMA-METAC (A), DMA-HEM-1h, DMAHEM-4h, and DMA-HEM-24h (B), DMA-METAC-HEM-1h, DMA-METAC-HEM-4h, and DMA-METAC-HEM-24h (C) microgels and free HEM (D). The peak at $1540 \mathrm{~cm}^{-1}$ and 1640 $\mathrm{cm}^{-1}$ are related to N-H biding vibration and $\mathrm{C}=\mathrm{O}$ stretching of amide groups, which exist in HEAA, APMA, and METAC functionalities in the microgels. The peak at $1053 \mathrm{~cm}^{-1}$ for $-\mathrm{OH}$, and $1440 \mathrm{~cm}^{-1}$ for $-\mathrm{CH}_{2}-$ are attributed to HEAA. The peaks at $1524 \mathrm{~cm}^{-1}$ (black dashed line, for benzene ring stretch) and $1113 \mathrm{~cm}^{-1}$ (green dashed line, for $-\mathrm{C}-\mathrm{OH}$ ) are attributed to DMA. The peak at $947 \mathrm{~cm}^{-1}$ (black arrow) corresponds to the quaternary ammonium of METAC groups. The peak at $1020 \mathrm{~cm}^{-1}$ (adsorbed $\mathrm{O}_{2}$ on the $\mathrm{Fe}-\mathrm{OH}$ site, red arrow) corresponds to HEM functionalities (B and C) in DMA-m and DMA-METAC microgels. This peak has appeared at $1040 \mathrm{~cm}^{-1}$ for free HEM (D). 


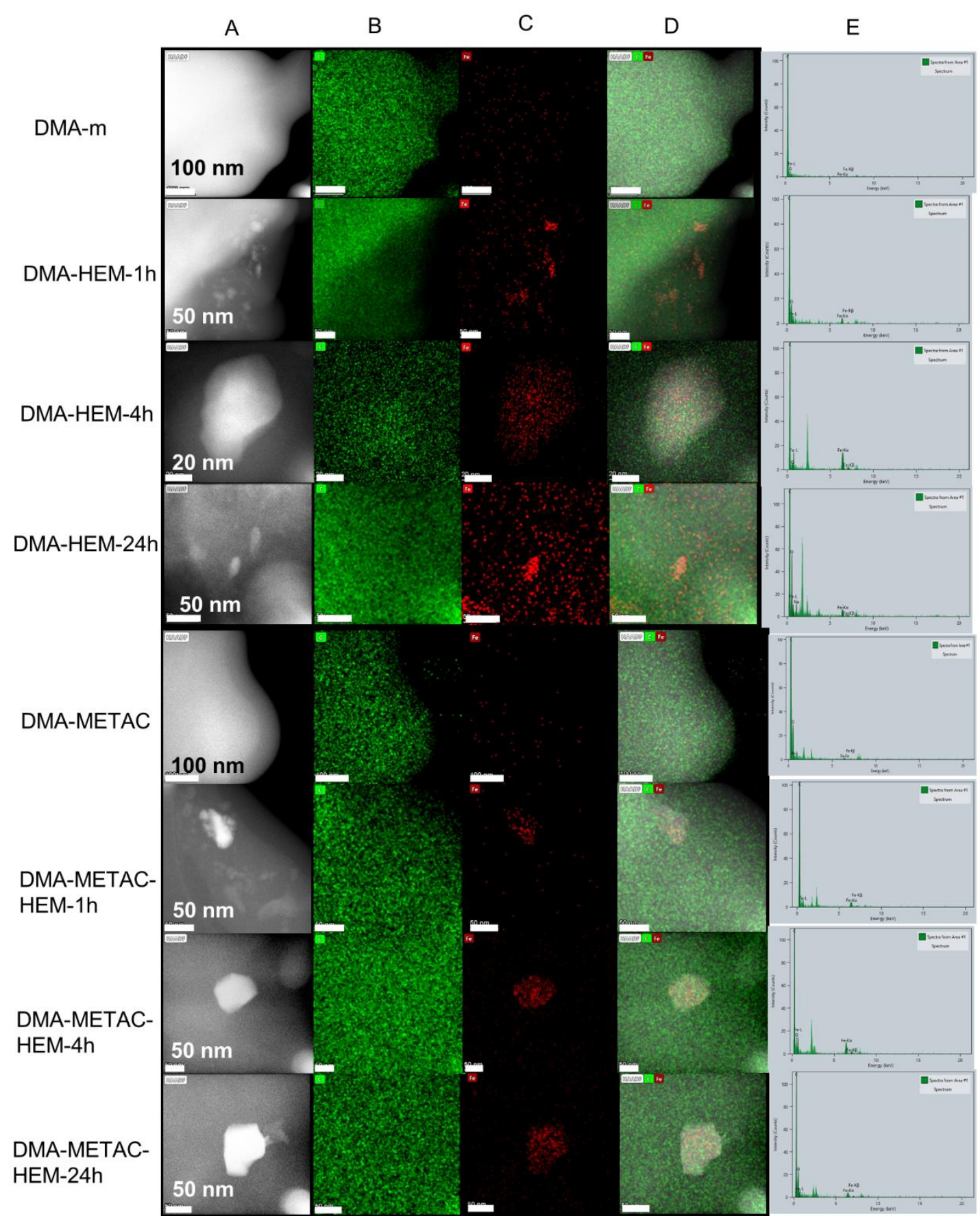

Figure S4. Cross-sectional STEM images (A) and corresponding EDX maps of elemental carbon (B), iron (C), and the overlapped image (D) of crashed DMA-m, DMA-HEM-1h, DMAHEM-4h, DMA-HEM-24h, DMA-METAC, DMA-METAC-HEM-1h, DMA-METAC-HEM4h and DMA-METAC-HEM-24h microgels. EDX spectrum showing carbon and iron peaks (E). 

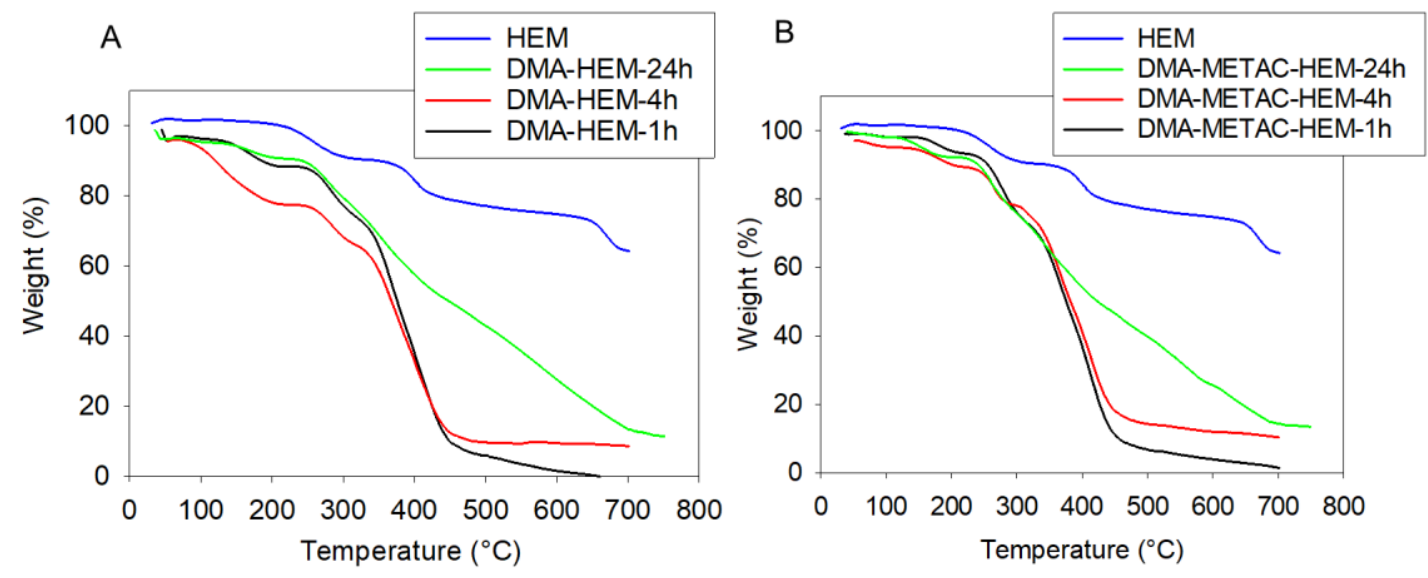

Figure S5. TGA plot of free HEM, DMA-HEM-1h, DMA-HEM-4h, and DMA-HEM-24h (A), as well as DMA-METAC-HEM-1h, DMA-METAC-HEM-4h and DMA-METAC-HEM-24h (B).

Table S1. Mass (\%) remained after thermal decomposition using TGA after heating the microgels to $700{ }^{\circ} \mathrm{C}$.

\begin{tabular}{|l|c|c|}
\hline \multicolumn{1}{|c|}{ Microgel } & $\begin{array}{c}\text { Remaining mass } \\
\text { of HEM (\%) }\end{array}$ & $\begin{array}{c}\text { Estimated Fe } \\
\text { content (\%)* }\end{array}$ \\
\hline HEM & 64.3 & $8.8^{\$}$ \\
\hline DMA-HEM-1h & 0 & 0 \\
\hline DMA-HEM-4h & 8.7 & 1.19 \\
\hline DMA-HEM-24h & 11.5 & 1.57 \\
\hline DMA-METAC-HEM-1h & 1.4 & 0.19 \\
\hline DMA-METAC-HEM-4h & 10.3 & 1.41 \\
\hline DMA-METAC0HEM-24h & 13.5 & 1.85 \\
\hline
\end{tabular}

* Estimated based on the remaining mass in HEM and the Fe content in the chemical structure of HEM

$\$$ Calculated Fe content based on the chemical structure of HEM

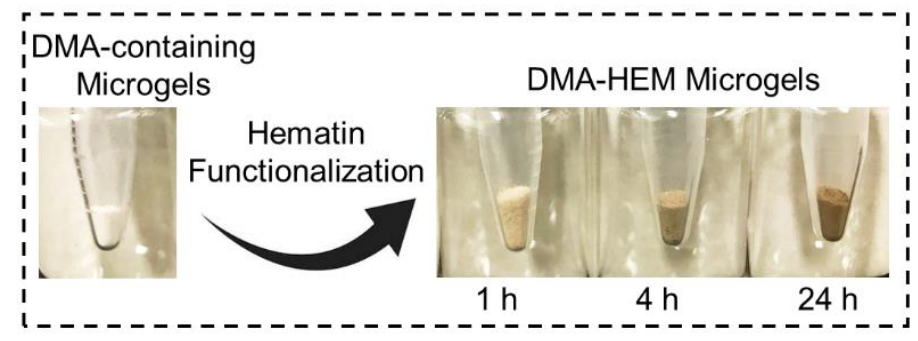

Figure S6. Effect of HEM-functionalization reaction time on the color of the microgels. 


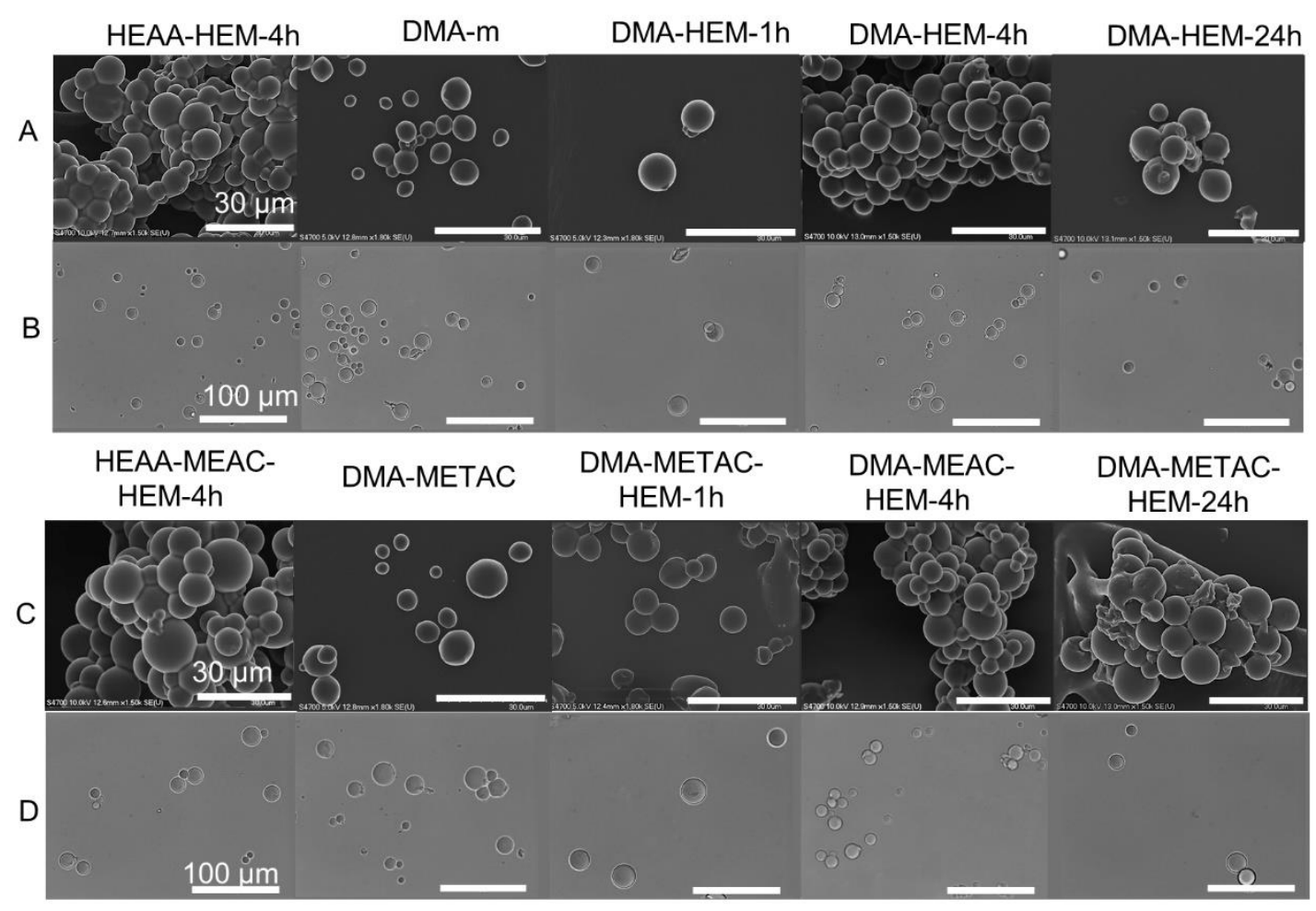

Figure S7. FESEM and phase contrast microscopy images of HEAA-HEM-4h, DMA-m, DMA-HEM-1h, DMA-HEM-4h, and DMA-HEM-24h (A and B, respectively), as well as HEAA-METAC-HEM-4h, DMA-METAC, DMA-METAC-HEM-1h, DMA-METAC-HEM-4h and DMA-METAC-HEM-24h (D and C, respectively).

Table S2. Average particle size of dried and hydrated microgels and their swelling ratios.

\begin{tabular}{|l|c|c|c|}
\hline Microgel Formulations & $\begin{array}{c}\text { Dried } \\
\text { Microgel } \\
(\boldsymbol{\mu m})\end{array}$ & $\begin{array}{c}\text { Equilibrated in } \\
\mathbf{p H} \text { 7.4 PBS } \\
(\boldsymbol{\mu m})\end{array}$ & $\begin{array}{c}\text { Swelling } \\
\text { ratio }\end{array}$ \\
\hline DMA-m & $8.1 \pm 2.9$ & $17.4 \pm 3.9$ & $10.5 \pm 1.3$ \\
\hline DMA-HEM-1h & $11.55 \pm 2.5$ & $22.3 \pm 2.4$ & $7.2 \pm 2.3$ \\
\hline DMA-HEM-4h & $10.2 \pm 2.1$ & $16.7 \pm 3.2$ & $4.0 \pm 2.5$ \\
\hline DMA-HEM-24h & $10 \pm 2.4$ & $13.6 \pm 2.5$ & $2.4 \pm 3.0$ \\
\hline DMA-METAC & $9.3 \pm 2.9$ & $20.5 \pm 2.5$ & $11 \pm 3.4$ \\
\hline DMA-METAC-HEM-1h & $10.63 \pm 1.9$ & $22.2 \pm 4.1$ & $7.4 \pm 4.8$ \\
\hline DMA-METAC-HEM-4h & $10 \pm 3.0$ & $19.5 \pm 4.4$ & $7.2 \pm 4.3$ \\
\hline DMA-METAC-HEM-24h & $12.2 \pm 2.9$ & $20.6 \pm 4.1$ & $4.4 \pm 3.1$ \\
\hline
\end{tabular}

Table S3. Zeta potential of microgels.

\begin{tabular}{|l|c|c|}
\hline \multicolumn{1}{|c|}{ Microgels } & Zeta potential $(\mathrm{mV})$ & $\mathrm{SD}$ \\
\hline DMA-m & 30.5 & 0.2 \\
\hline DMA-METAC & 33.1 & 0.6 \\
\hline
\end{tabular}




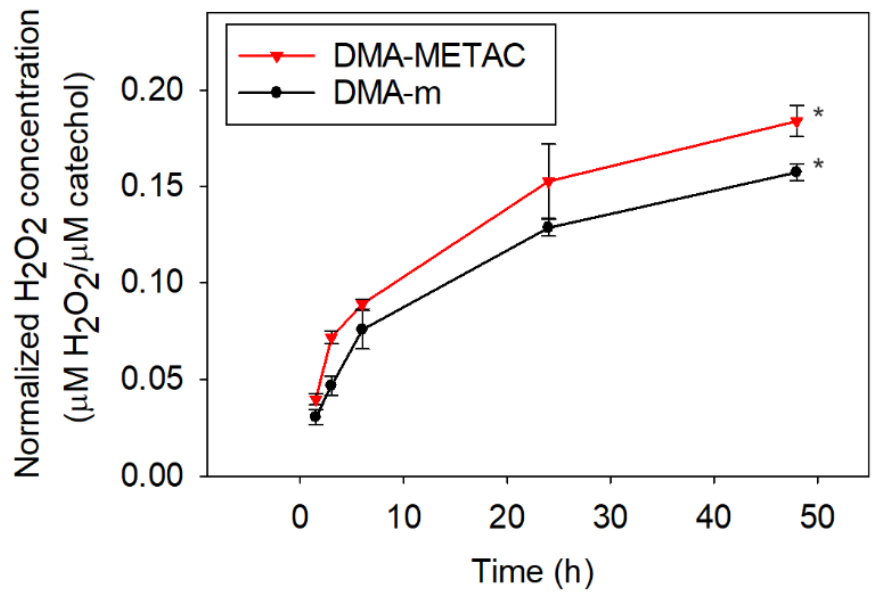

Figure S8. $\mathrm{H}_{2} \mathrm{O}_{2}$ concentration normalized to the catechol concentration generated from microgels. ${ }^{*} \mathrm{p}<0.05$ when compared to the other microgels at the same time point.

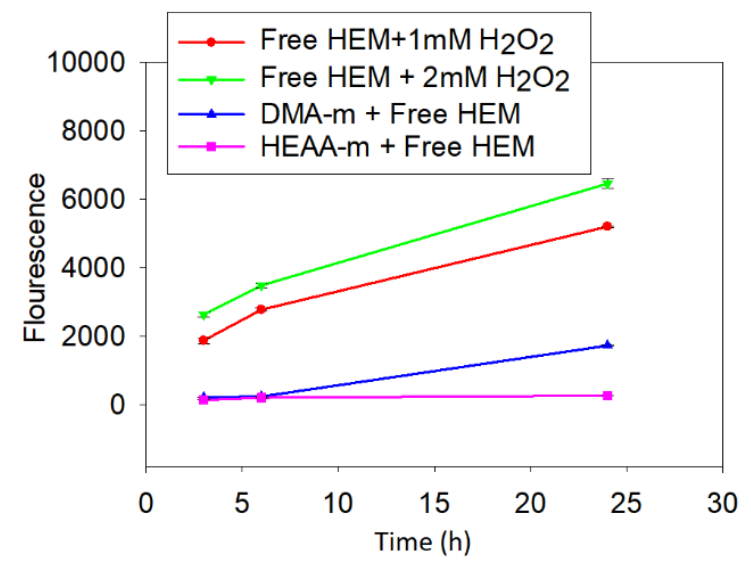

Figure S9. ${ }^{\circ} \mathrm{OH}$ detection using HPF probe from free HEM in the presence of 1-2 $\mathrm{mM} \mathrm{H}_{2} \mathrm{O}_{2}$, $25 \mathrm{mg}$ of DMA-m and HEAA-m microgels.
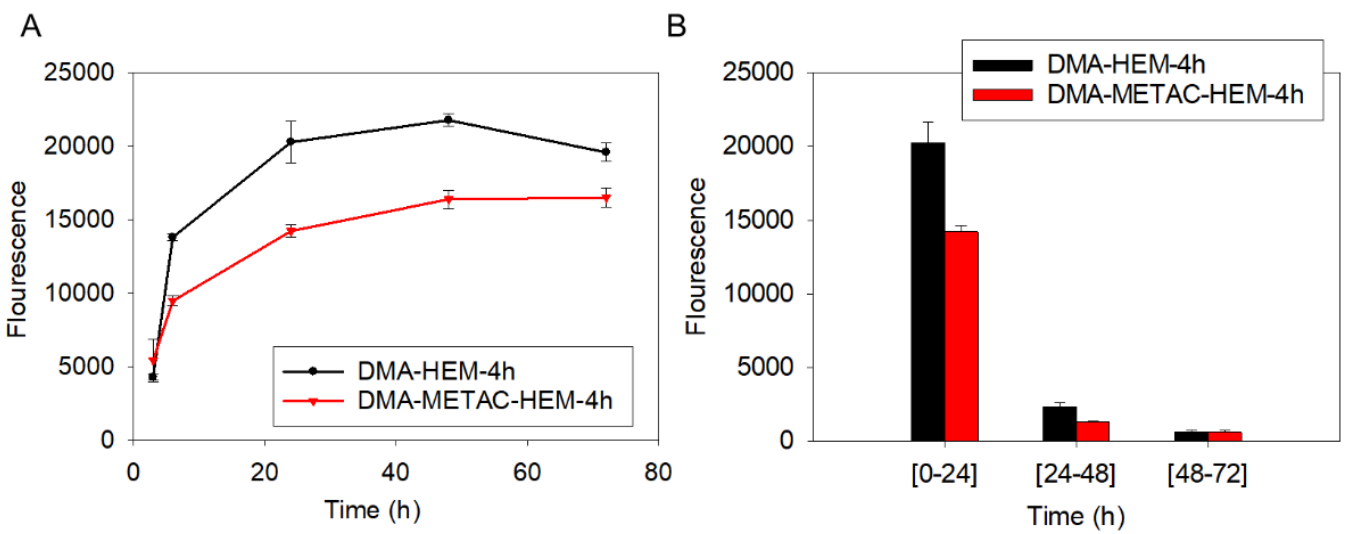

Figure S10. ${ }^{\circ} \mathrm{OH}$ detection using HPF probe from microgels. Cumulative fluorescence intensity detected for up to $72 \mathrm{~h}$ (A), and discrete fluorescence intensity detected in a given 24 $\mathrm{h}$ period after the start of microgel hydration (B). 


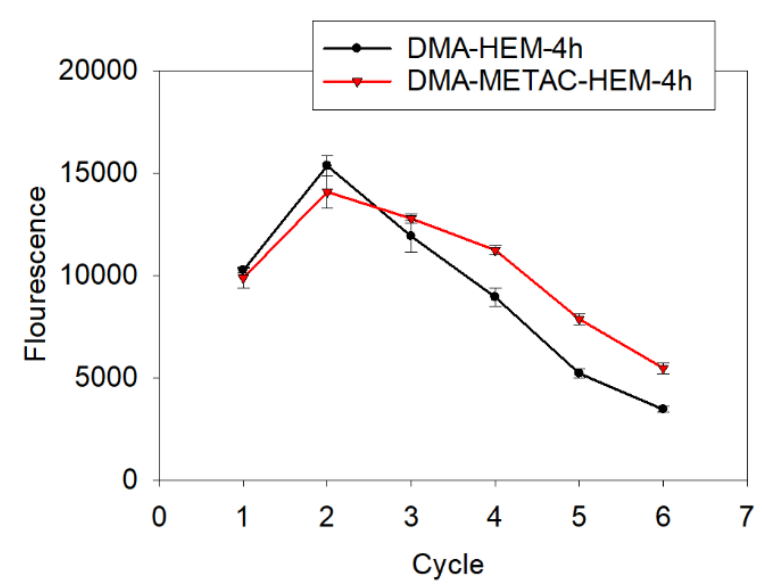

Figure S11. ${ }^{\circ} \mathrm{OH}$ detection using HPF probe from microgels when repeatedly incubated at $\mathrm{pH}$ 7.4 $\mathrm{PB}$ at room temperature for $12 \mathrm{~h}$ and washed with $\mathrm{pH} 3.5 \mathrm{DI}$ water.
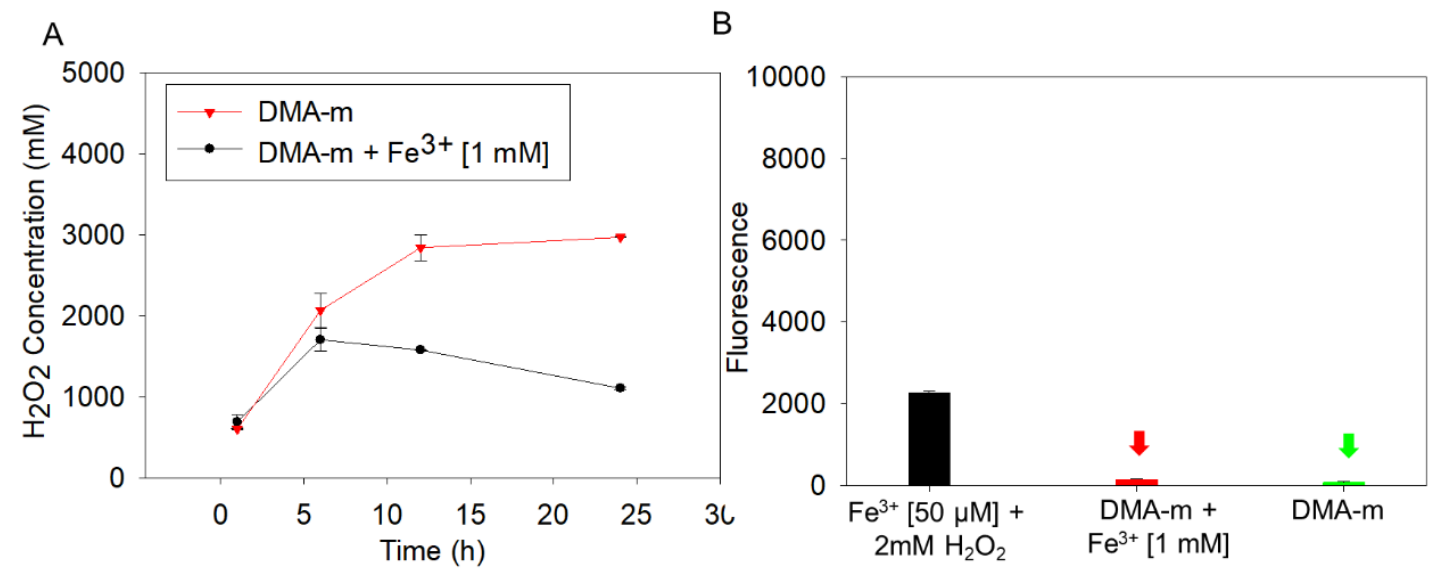

Figure S12. $\mathrm{H}_{2} \mathrm{O}_{2}$ generation from DMA-m microgels with and without free $\mathrm{Fe}^{3+}$ ions (A). $\cdot \mathrm{OH}$ detection using HPF probe from $2 \mathrm{mM} \mathrm{H}_{2} \mathrm{O}_{2}$, methyl catechol $(10 \mu \mathrm{M})$ and free $\mathrm{Fe}^{3+}$ ions (black bar), $25 \mathrm{mg}$ DMA-m in the presence of free $\mathrm{Fe}^{3+}$ ions (red bar, red arrow), and $25 \mathrm{mg}$ DMA-m (green bar, green arrow) (B). 


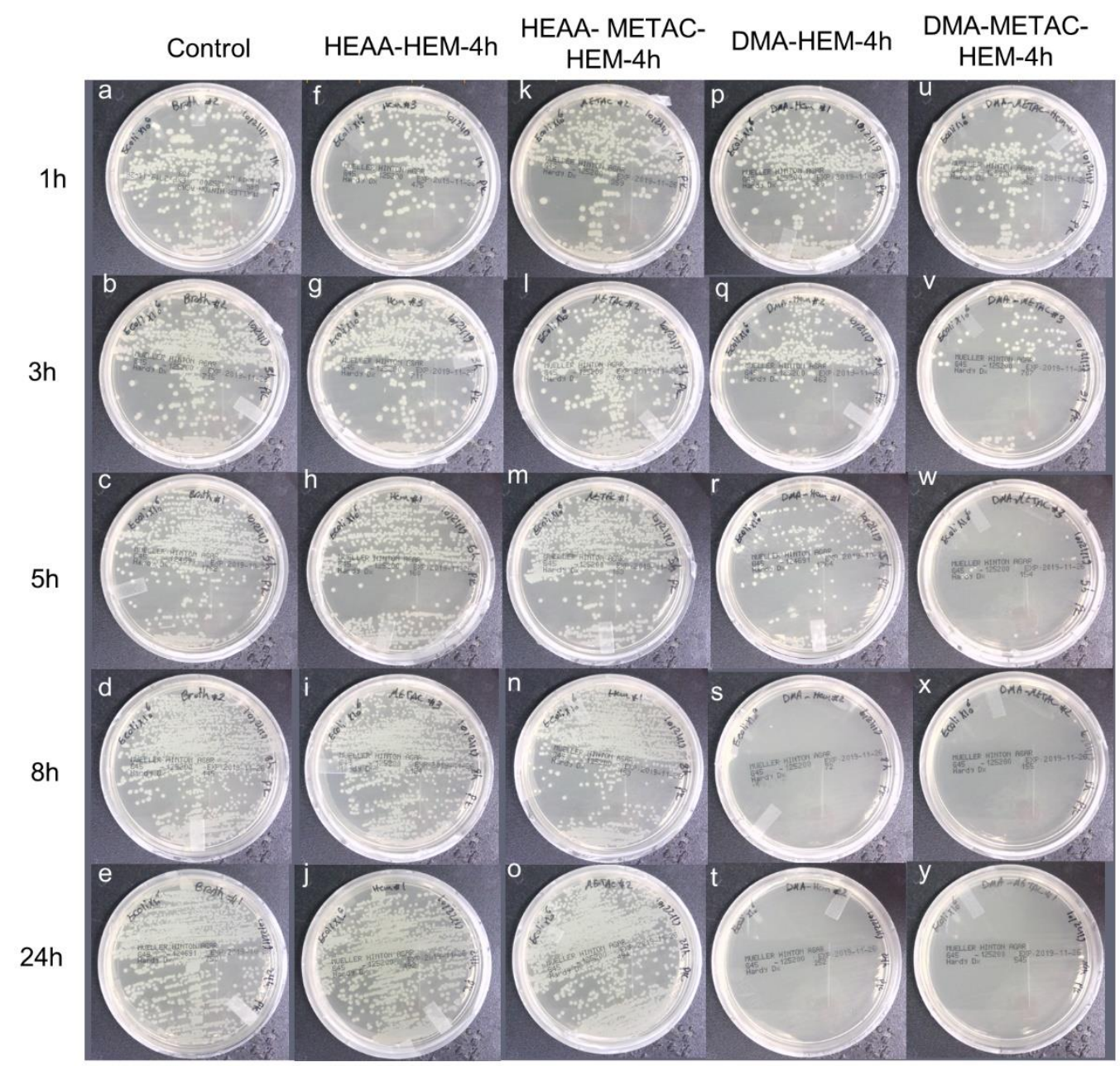

Figure S13. Photograph of the test plates with E. coli colonies (with initial concentration of $10^{6} \mathrm{cfu} / \mathrm{ml}$ ) exposed to PBS (no microgels, a-e), HEAA-HEM-4h (f-j), HEAA-METACHEM-4h (k-o), DMA-HEM-4h (p-q), and DMA-METAC-HEM-4h (u-y) microgels, taken at different incubation time. 


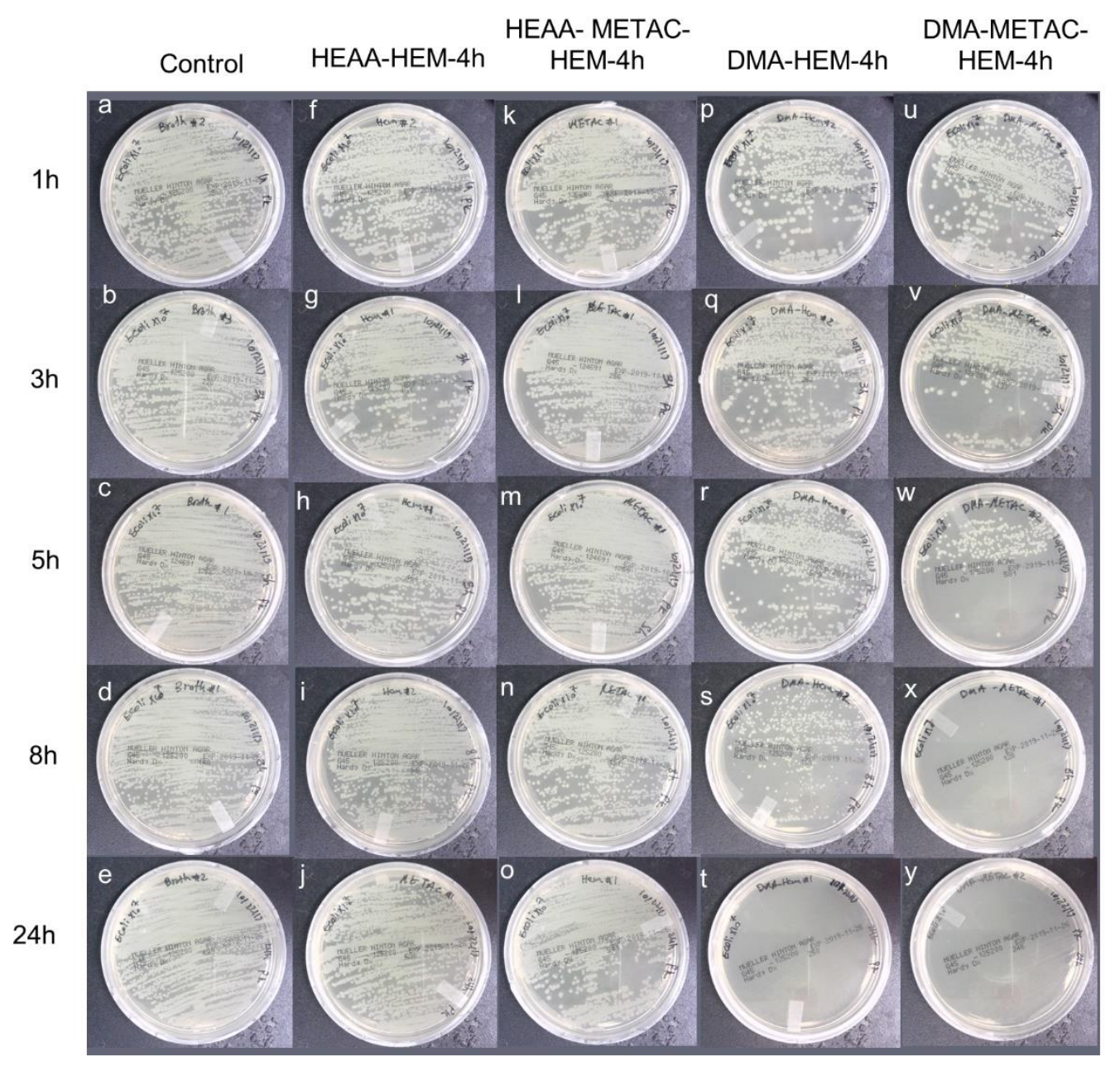

Figure S14. Photograph of the test plates with E. coli colonies (with initial concentration of $10^{7}$ $\mathrm{cfu} / \mathrm{ml}$ ) exposed to PBS (no microgels, a-e), HEAA-HEM-4h (f-j), HEAA-METAC-HEM-4h (k-o), DMA-HEM-4h (p-t), and DMA-METAC-HEM-4h (u-y) microgels, taken at different incubation time. 


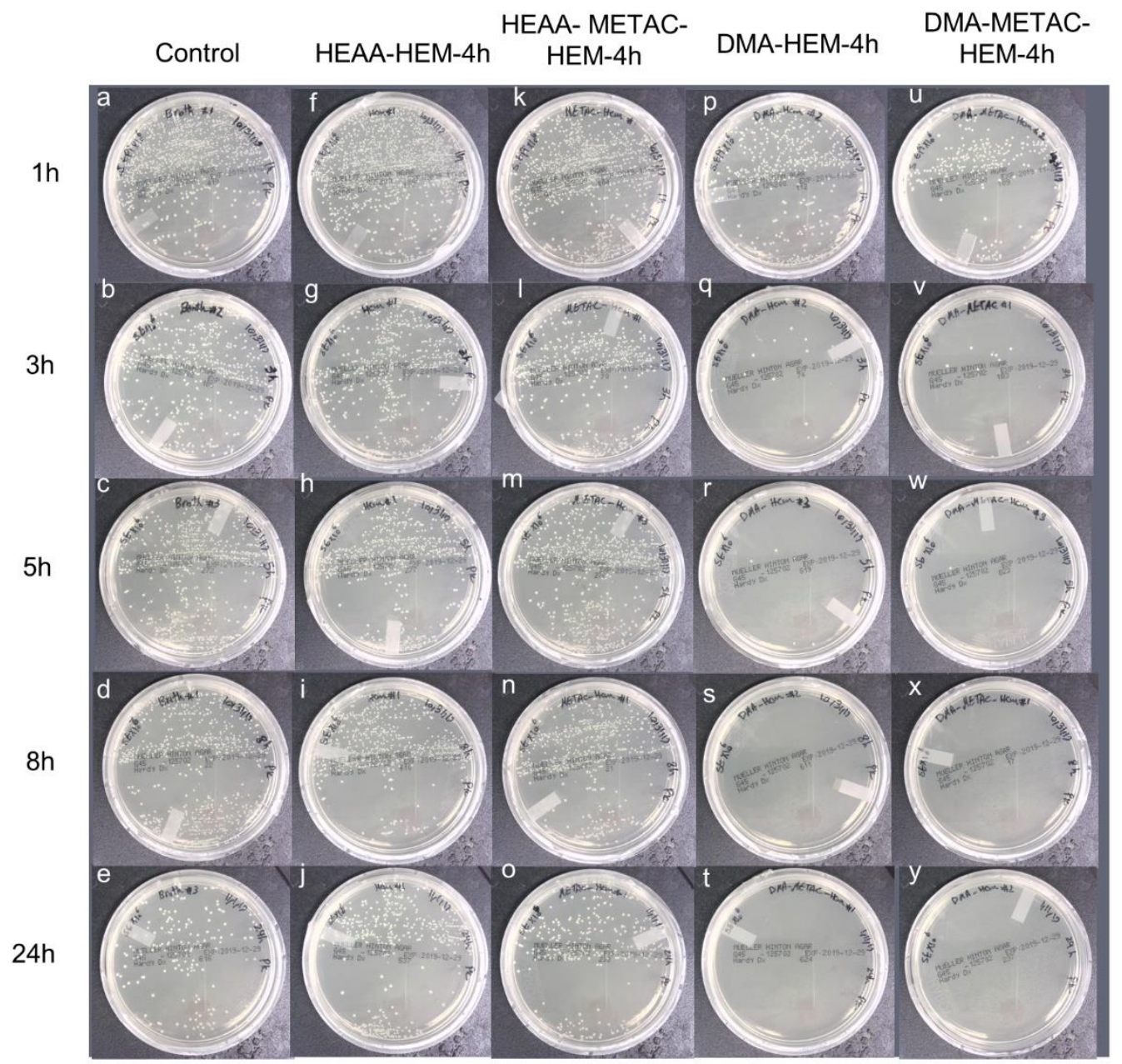

Figure S15. Photograph of the test plates with S. epidermidis colonies (with initial concentration of $10^{6} \mathrm{cfu} / \mathrm{ml}$ ) exposed to PBS (no microgels, a-e), HEAA-HEM-4h (f-j), HEAAMETAC-HEM-4h (k-o), DMA-HEM-4h (p-t), and DMA-METAC-HEM-4h (u-y) microgels, taken at different incubation time. 


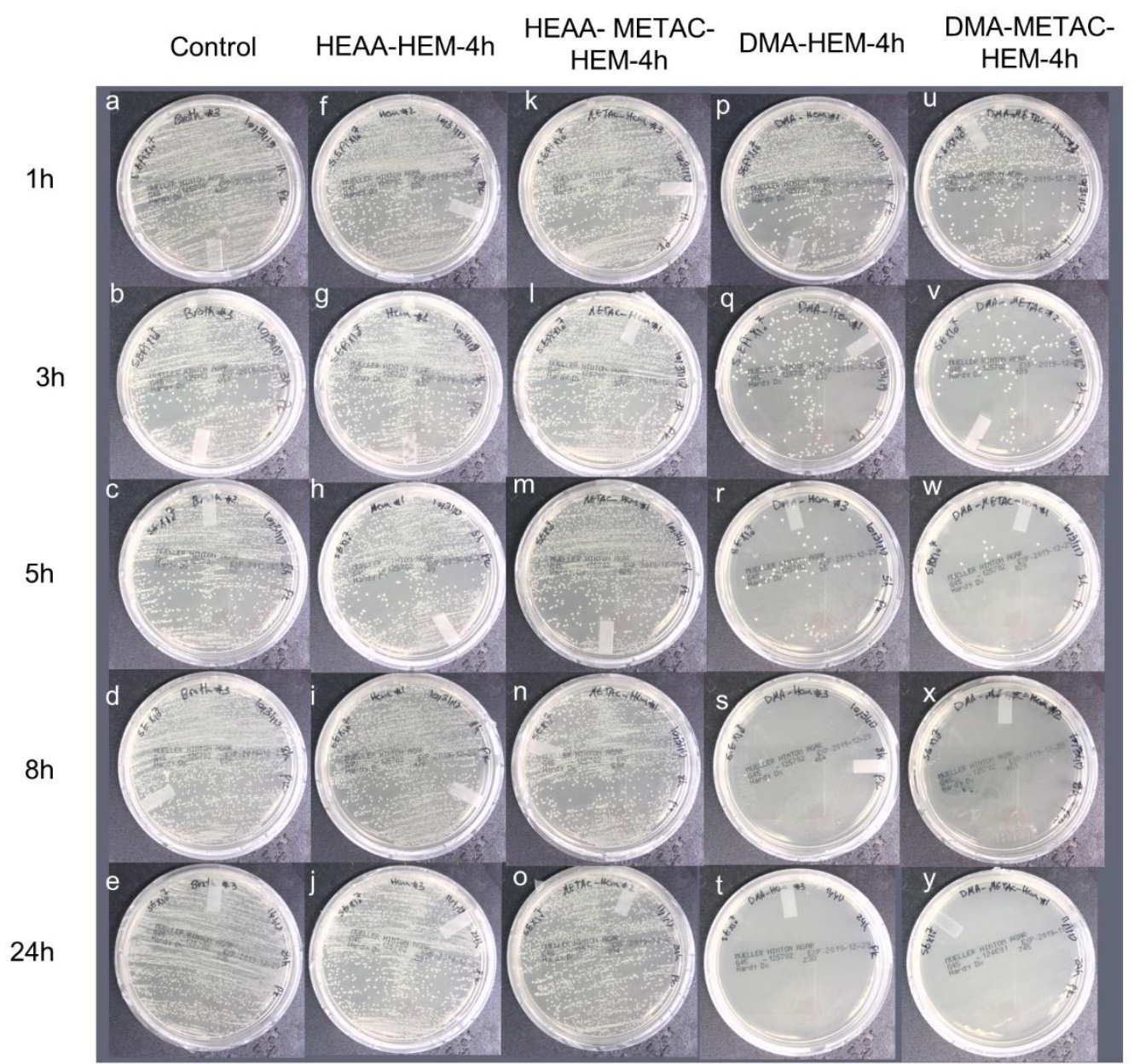

Figure S16. Photograph of the test plates with $S$. epidermidis colonies (with initial concentration of $10^{7} \mathrm{cfu} / \mathrm{ml}$ exposed to PBS (no microgels, a-e), HEAA-HEM-4h (f-j), HEAAMETAC-HEM-4h (k-o), DMA-HEM-4h (p-t), and DMA-METAC-HEM-4h (u-y) microgels, taken at different incubation time. 
A

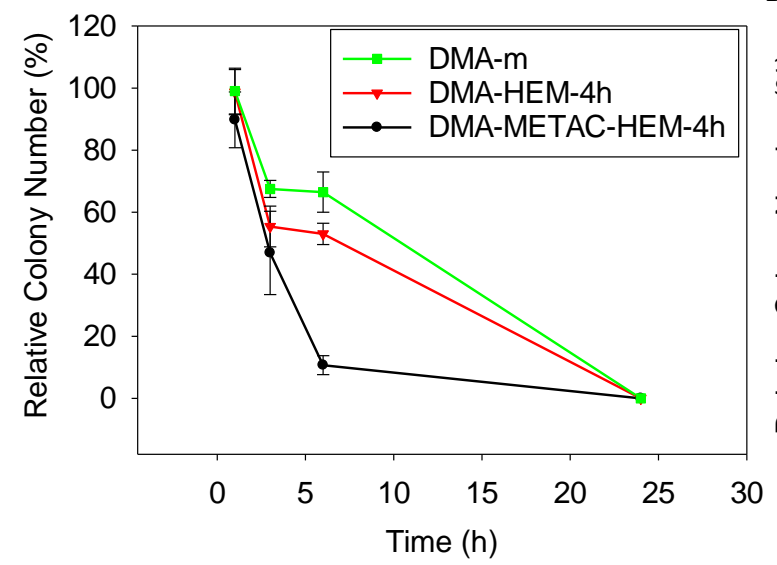

B

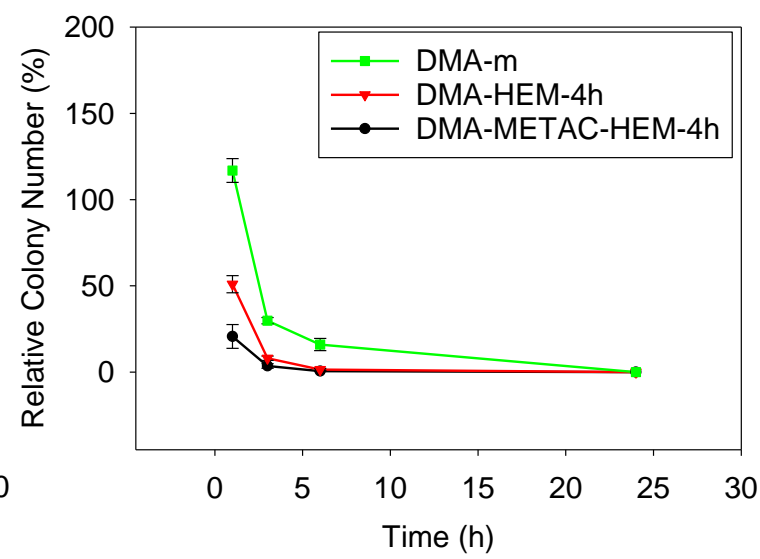

Figure S17. Relative bacteria colony number for E. coli and S. epidermidis (with the initial concentration of $10^{7} \mathrm{cfu} / \mathrm{ml}, \mathrm{A}$ and $\mathrm{B}$, respectively) incubated with different microgel formulations.

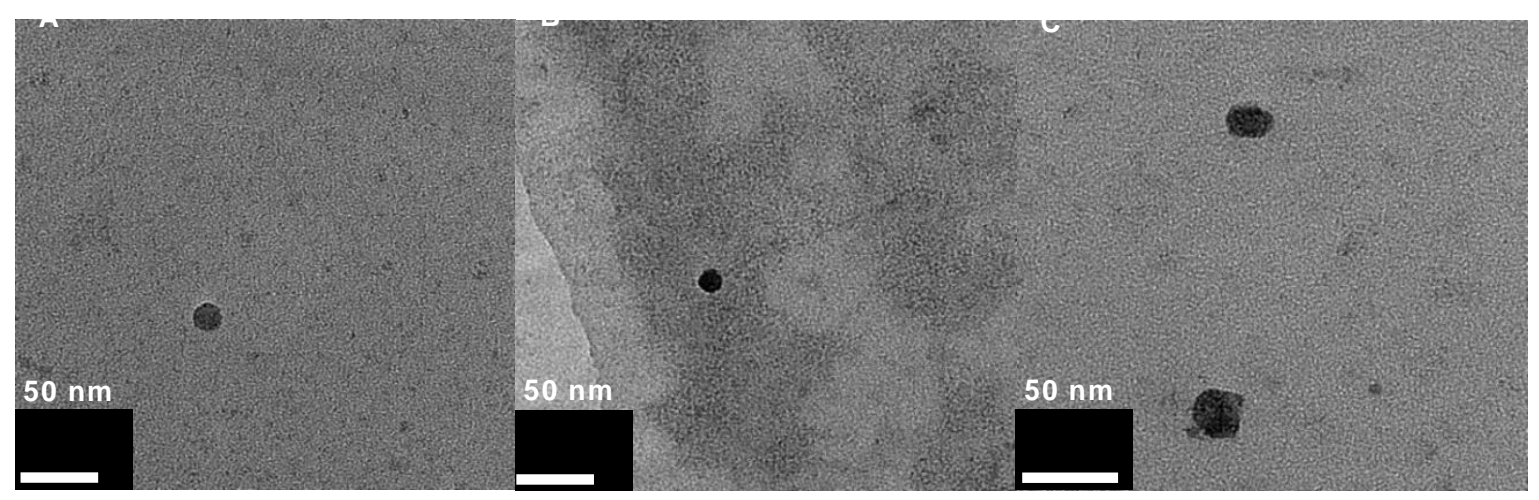

Figure S18. STEM images of PPV before (A) and after treatment with DMA-m (B) and DMAMETAC-HEM-4h (C) microgels.

Table S4. Size distribution of PPV before and after exposure to microgels*,\$

\begin{tabular}{|l|c|c|c|}
\hline & untreated & DMA-m & DMA-METAC-HEM-4h \\
\hline Particle size $(\mathrm{nm})$ & $20.8 \pm 1.5$ & $20.5 \pm 6.3$ & $23.6 \pm 3.7$ \\
\hline
\end{tabular}

* Based on the measurement of 6 virus particles using from at least 3 TEM images.

${ }^{\$}$ Values were not statistically different between groups. 
Table S5. Values used to calculate the mesh size $(\xi)$ of microgels.

\begin{tabular}{|l|c|c|c|}
\hline Microgel & $v_{\mathrm{s}}$ & $\begin{array}{c}\bar{M}_{C} \\
(\mathrm{kDa})\end{array}$ & $\xi(\mathrm{nm})$ \\
\hline HEAA-HEM-4h & 0.4 & 0.5 & 1.9 \\
\hline DMA-HEM-4h & 0.2 & 3.9 & 6.3 \\
\hline HEAA-METAC-HEM-4h & 0.1 & 2.5 & 4.7 \\
\hline DMA-METAC-HEM-4h & 0.1 & 15.6 & 14.8 \\
\hline
\end{tabular}

